\title{
Estado nutricional de pacientes infectados por el virus de la inmunodeficiencia humana (VIH)
}

\author{
D. A. DE LUIS*,**, P. BACHILLER**, O. IZAOLA***, J. M. EIROS BOUZA**, \\ R. ALLER $* * * * * *$
}

*Sección de Endocrinología y Nutrición Clínica. Hospital del Río Hortega. **Instituto de Endocrinología y Nutrición Clínica. Facultad de Medicina. ***Servicio Aparato Digestivo. Hospital Clínico Universitario. Valladolid

\author{
NUTRITIONAL STATE IN HIV INFECTED PATIENTS
}

\section{RESUMEN}

Objetivos: La valoración del estado nutricional en los pacientes VIH debe ser un pilar fundamental en el tratamiento integral de estos enfermos. El objetivo de nuestro trabajo es estudiar el estado nutricional mediante parámetros bioquímicos y antropométricos de un grupo de pacientes con infección por virus de la inmunodeficiencia humana con tratamiento antiretroviral.

Pacientes y métodos: Se valoraron bioquímicamente y antropométricamente un total de 119 pacientes VIH positivos. A todos los pacientes se les tomaron los siguientes datos epidemiológicos; edad, sexo, tratamiento con fármacos anti-retrovirales, infección por VIH, realizándose una valoración antropométrica (peso, talla, pliegue tricipital (PT), circunferencia braquial (CB), circunferencia muscular del brazo (CMB), índice de masa corporal (IMC) y una valoración bioquímica nutricional (albúmina, prealbúmina, transferrina, proteínas totales, triglicéridos, colesterol total, linfocitos totales y recuento de CD4).

Resultados: Los pacientes presentaron una media de edad de $37,9 \pm 9,9$ años, peso $64,5 \pm 13,2 \mathrm{~kg}$ y el índice de masa corporal $22,5 \pm 3,5$. Los valores de proteínas totales, albúmina, prealbúmina y transferrina estaban dentro de la normalidad, solo en los pacientes con estadio SIDA se detectaron niveles de transferrina inferiores al resto de los estadíos $(262,5 \pm 49,2 \mathrm{mg} / \mathrm{dl}$ vs $277,8 \pm 87,5 ; \mathrm{p}<0,05)$. La distribución de percentiles de los parámetros antropométricos mostró una depleción del compartimento proteico muscular (CB y CMB), el 53,1\% de los pacientes presentaba un percentil del PT inferior o igual al P 50, un $91,8 \%$ presentaban un percentil de CB inferior o igual al P 50, al analizar la CMB un $91,8 \%$ de los pacientes tenían un percentil inferior o igual al P 50. La hipertrigliceridemia estuvo presente en un $30,5 \%$ de los pacientes, relacionándose con el estadio VIH y el número de fármacos antiretrovirales que tomaba el paciente.

Conclusión: El estado nutricional de los pacientes VIH en nuestro estudio es bueno presentando solo una reducción del compartimento proteico muscular. La transferrina se ha mostrado como un parámetro nutricional afectado en los estadios avanzados. La hipertrigliceridemia existente en estos pacientes parece secundaria al tratamiento antiretroviral.

PALABRAS CLAVE: Antropometría. Bioquímica. Nutrición. VIH.

\section{ABSTRACT}

Objectives: Nutritional assessment in HIV infected patients must be a main objetive in the treatment of this disease. The main objetive of our work was to study the nutritional status with biochemical and anthropo metrical parameters in a group of HIV infected patients.

Material and methods: A total of 119 patients were evaluated. In all patients the next parameters were assessed: age, sex, treatment with anti-retroviral drugs, performed an anthropometric evaluation (weight, height, tricipital skinfold, arm circumference, arm muscular circumfe rence and body mass index) and biochemical evaluation (albumin, pre albumin, transferrin, total proteins, triglycerides, cholesterol, lymphoci tes and count of $C D 4$ ).

Results: Patients had an average age $37.9 \pm 9.9$ years, weight $64.5 \pm 13.2 \mathrm{~kg}$ and body mass index $22.5 \pm 3.5$. The values of total proteins, albumin, prealbumin y transferrin were all normal, only patients with AIDS had low levels of transferrin $(262.5 \pm 49.2 \mathrm{mg} / \mathrm{dl}$ vs $277.8 \pm 87.5$; $p<0.05)$. Percentil distribution of anthropometric parameters showed a deep depletion in muscular protein compartment, $53.1 \%$ of patients had skinfold under $P$ 50, 91.8\% had arm circunference under $P$ 50, and $91.8 \%$ arm muscular circunference under P 50. High levels of triglyce rides were detected in $30.5 \%$, these levels are related with the HIV pha se and the number of antiretroviral drugs.

Conclusion: Nutritional status in HIV infected patients is good in our population, only a deep depletion in muscular proteic compartment was detected. Transferrin was decreased in AIDS patients. High levels of triglycerides were secondary to the number of antiretroviral drug taking by patients.

KEY WORDS: Anthropometry. Bioquimic. Nutrition. HIV.

De Luis DA, Bachiller P, Izaola O, Eiros Bouza JM, Aller R. Estado nutricional de pacientes infectados por el virus de la inmunodeficiencia humana (VIH). An Med Interna (Madrid) 2001; 18: 619-623.

Trabajo aceptado: 2 de Julio de 2001

Correspondencia: Daniel de Luis Román. Endocrinología y Nutrición. Facultad de Medicina. Hospital Universitario Río Hortega. C/ Los Perales 16, Urb. Las Aceñas. 47130 Valladolid 


\section{INTRODUCCIÓN}

Aunque el pronóstico de los pacientes con infección por VIH ha mejorado desde la introducción de nuevas terapias, encabezadas por los inhibidores de la proteasa, la situación nutricional de los pacientes VIH sigue siendo un problema clínico en esta población con peculiaridades nuevas, en parte secundarias a las nuevas terapias antiretrovirales (1).

En ocasiones en nuestra práctica clínica diaria un paciente con infección por VIH esta recibiendo un tratamiento multidisciplinar correcto, sin prestar atención a su situación nutricional que condiciona sin duda la situación clínica y el pronóstico de morbi-mortalidad (2). Por tanto es necesario tener en cuenta que la valoración y el soporte nutricional debe ser uno de los primeros escalones en el tratamiento de este tipo de pacientes (3). Para valorar el estado nutricional disponemos de parámetros antropométricos y de parámetros bioquímicos, muchos de ellos al alcance de cualquier consulta y que nos permitirán clasificar a nuestros pacientes en función de su situación nutricional.

El objetivo de nuestro trabajo es estudiar el estado nutricional mediante estos parámetros de un grupo de pacientes con infección por virus de la inmunodeficiencia humana en tratamiento con fármacos antiretrovirales.

\section{MATERIAL Y MÉTODOS}

\section{PACIENTES}

Se estudiaron un total de 119 pacientes con infección por VIH que acudieron a nuestra consulta para realizar una valoración nutricional y prescribir el correspondiente tratamiento nutricional, desde enero a junio del 2000. Estos pacientes procedían de una área rural y urbana de Valladolid. A todos los pacientes se les tomaron los siguientes datos epidemiológicos; edad, sexo, tratamiento con fármacos anti-retrovirales y estadío de la infección VIH, realizandose una valoración antropométrica y una valoración bioquímica nutricional.

\section{VALORACIÓN ANTROPOMÉTRICA}

Se midió en todos ellos el pliegue tricipital (PT). Para medirlo se mantuvo al paciente en bipedestación y con su brazo no dominante colgado libremente. A continuación se localizó en la cara posterior el punto medio entre el acromion y el olecranon, por encima del triceps. Se pellizcó suavemente la piel y el tejido celular subcutáneo en este punto y se aplicó un calibrador regulado a presión $\left(10 \mathrm{~g} / \mathrm{mm}^{2}\right)$ durante 3 segundos (tipo Langer). La medición se realizó 3 veces y se tomó la media de estas, expresado en $\mathrm{mm}$.

También se determinó la circunferencia del brazo (CB) y la circunferencia muscular del brazo (CMB). Para determinar estos parámetros se midió la $\mathrm{CB}$ con una cinta métrica calibrada en milímetros en el mismo lugar del brazo donde se realizó la medición del PT, la medición se realizó tres veces y se tomó la media. Para el cálculo final de la CMB se utilizó la fórmula $(\mathrm{CMB}=\mathrm{CB}-(\mathrm{PT} \times \mathrm{x}, 314))$, expresado en $\mathrm{cm}$.

A todos se les determinó el peso, peso ideal, talla e índice de masa corporal $(\mathrm{IMC})=$ peso $(\mathrm{kg}) / \mathrm{talla}^{2}(\mathrm{~m})$.

Todos estos parámetros antropométricos fueron medidos siempre por la misma persona, para evitar la variabilidad interindividual en las medidas antropométricas. Se utilizaron como tablas de referencia las de Frisancho y cols. (4).

\section{VALORACIÓN BIOQUÍMICA}

Se determinaron los siguientes parámetros (valores normales) con un autoanalizador (Hitachi, ATM), glucosa (75$110 \mathrm{mg} / \mathrm{dl})$, urea $(10-50 \mathrm{mg} / \mathrm{dl})$, colesterol total $(110-200$ $\mathrm{mg} / \mathrm{dl})$, triglicéridos $(50-200 \mathrm{mg} / \mathrm{dl})$, creatinina $(0,6-1,1$ $\mathrm{mg} / \mathrm{dl})$, proteínas totales $(6,6-8,7 \mathrm{gr} / \mathrm{dl})$, albúmina $(3,5-4,5$ $\mathrm{gr} / \mathrm{dl})$, prealbúmina $(18-28 \mathrm{mg} / \mathrm{dl})$, transferrina $(250-350$ $\mathrm{mg} / \mathrm{dl})$, linfocitos $\left(1,2-3,5,10^{3} / \mathrm{uL}\right)$ y CD4 (contaje/uL).

\section{ANÁLISIS ESTADÍSTICO}

Con los datos obtenidos se abrió una base de datos con el paquete estadístico (SPSS, Inc., IL., USA). Se realizó un análisis descriptivo de las variables cuantitativas, expresadas como (media + desviación estándar). También se realizó una distribución de las variables en percentiles para demostrar el grado de desviación sobre la normalidad de estos pacientes. Se realizó correlación de Pearson y Spearman en función de las variables analizadas. En el análisis de variables cuantitativas pertenecientes a más de dos grupos se utilizó el análisis de ANOVA.

\section{RESULTADOS}

Se estudiaron un total de 119 pacientes (88 varones y 31 mujeres), la edad media fue $37,9+9,9$ años, el peso $64,5+13,2$ $\mathrm{kg}$ y el índice de masa corporal $22,5+3,5$. Todos los pacientes estaban recibiendo tratamiento antiretroviral en el momento del estudio nutricional, un 4,9\% recibían 2 fármacos, un $75,5 \% 3$ fármacos y un 19,6\% 4 fármacos. Un 25,8\% pertenecían al estadio A, un $41,3 \%$ al estadio B y un $32,9 \%$ al C.

La valoración bioquímica del grupo total se muestra en la tabla I, mostrando unos valores medios de proteínas viscerales dentro de la normalidad, aunque un alto porcentaje de pacientes presentaron alteraciones en el patrón lipídico con niveles

\section{TABLA I}

VALORACIÓN BIOQUÍM ICA PACIENTES INFECCIÓN VIH

\begin{tabular}{lc}
\hline Parámetro & $\begin{array}{c}\text { M edia } \\
\text { (desviación estándar) }\end{array}$ \\
\hline Glucosa $(\mathrm{mg} / \mathrm{dl})$ & $98,1 \pm 32,5$ \\
Urea $(\mathrm{mg} / \mathrm{dl})$ & $38 \pm 35,8$ \\
Colesterol total $(\mathrm{mg} / \mathrm{dl})$ & $193,4 \pm 51,9$ \\
Triglicéridos $(\mathrm{mg} / \mathrm{dl})$ & $198,7 \pm 38,4$ \\
Creatinina $(\mathrm{mg} / \mathrm{dl})$ & $0,8 \pm 0,3$ \\
Proteínas totales $(\mathrm{g} / \mathrm{dl})$ & $7,81 \pm 0,6$ \\
Albúmina $(\mathrm{g} / \mathrm{dl})$ & $4,1 \pm 0,73$ \\
Prealbúmina $(\mathrm{mg} / \mathrm{dl})$ & $23,3 \pm 7,2$ \\
Transferrina $(\mathrm{mg} / \mathrm{dl})$ & $250,7 \pm 59,5$ \\
Linfocitos $(103 / \mathrm{uL})$ & $2.920 \pm 4.970$ \\
CD4 (recuento $/ \mathrm{uL})$ & $594,7 \pm 512,8$ \\
\hline
\end{tabular}


de triglicéridos por encima de $200 \mathrm{mg} / \mathrm{dl}$ en un $30,5 \%$ y de colesterol por encima de $200 \mathrm{mg} / \mathrm{dl}$ en un $38,1 \%$, las alteraciones del metabolismo de los hidratos de carbono se detectaron en un 4,8\% de los pacientes con glucemias por encima de 126 $\mathrm{mg} / \mathrm{dl}$ y un $11,4 \%$ por encima de $110 \mathrm{mg} / \mathrm{dl}$, parámetro que actualmente se considera como alteración de la glucemia en ayunas.

La valoración antropométrica mostró la circunferencia muscular del brazo y la circunferencia del brazo por debajo de la media, sin embargo el pliegue tricipital, el peso y el IMC estaban dentro de la normalidad (Tabla II). Al realizar una distribución de estos tres parámetros antropométricos en función de los percentiles descritos en las tablas de referencia (4), se detectó como el 53,1\% de los pacientes presentaba un percentil del PT inferior o igual al P 50, un 91,8\% presentaban un percentil de $\mathrm{CB}$ inferior o igual al $\mathrm{P} 50$, al analizar la CMB un $91,8 \%$ de los pacientes tenían un percentil inferior o igual al P 50 (Tabla III). Estos datos indicaban una severa reducción del compartimento proteico muscular, con conservación del compartimento graso.

TABLA ॥

\begin{tabular}{lc}
\multicolumn{2}{c}{ TABLA II } \\
\multicolumn{2}{c}{ VALO RACIÓ N ANTRO PO M ÉTRICA DE PACIENTES } \\
\multicolumn{2}{c}{ TUM O RALES } \\
\hline \multicolumn{3}{c}{ Media (desviación estándar) } \\
\hline IM C & $22,5 \pm 3,5$ \\
PESO (kg) & $64,5 \pm 13,2$ \\
PT (mm) & $19,1 \pm 4,7$ \\
CB (cm) & $27,4 \pm 3,7$ \\
CM B $(\mathrm{cm})$ & $21,5 \pm 3,8$
\end{tabular}

IMC (índice de masa corporal), PT (pliegue tricipital), CB (circunferencia braquial), CM B (circunferencia muscular del brazo). No existieron diferencias significativas en estos parámetros en los diferentes estadios.

TABLA III

DISTRIBUCIÓN DE PERCENTILES DE VALORES ANTRO PO MÉTRICOS SEGÚN TABLA DE REFERENCIA DE FRISANCHO Y COLS. (4)

\begin{tabular}{lccc}
\hline & $\begin{array}{c}\text { P. tricipital } \\
(\%)\end{array}$ & $\begin{array}{c}\text { C. brazo } \\
(\%)\end{array}$ & $\begin{array}{c}\text { C. muscular del brazo } \\
(\%)\end{array}$ \\
\hline P $<5$ & $4 \%$ & $50 \%$ & $74,1 \%$ \\
P 10 & $14,5 \%$ & $16,9 \%$ & $8,9 \%$ \\
P 25 & $17,7 \%$ & $16,9 \%$ & $4,8 \%$ \\
P 50 & $16,9 \%$ & $8 \%$ & $4 \%$ \\
P 75 & $32,2 \%$ & $0,8 \%$ & $2,4 \%$ \\
P 90 & $9,3 \%$ & $4,2 \%$ & $7,4 \%$ \\
P 95 & $5,4 \%$ & $3,2 \%$ & $0,8 \%$ \\
\hline
\end{tabular}

(\%): Porcentaje de pacientes en cada percentil.

Se realizó una distribución de las variables bioquímicas y antropométricas nutricionales de la población de pacientes VIH en percentiles (P25, P50, P75). La distribución de percentiles de los valores bioquímicos se muestra en la tabla IV presentando la albúmina, prealbúmina y transferrina unos percentiles 50 dentro de la normalidad. En la distribución por percentiles de los valores antropométricos (Tabla V), la cir-
TABLA IV

DISTRIBUCIÓ N DE PERCENTILES DE VALORES BIO Q UÍMICOS NUTRICIO NALES EN POBLACIÓN DEL ESTUDIO

\begin{tabular}{lccc}
\hline & $\mathrm{P}, 25$ & $\mathrm{P}, 50$ & $\mathrm{P}, 75$ \\
\hline Proteína $(\mathrm{g} / \mathrm{dl})$ & 7,4 & 7,8 & 8,1 \\
Albúmina $(\mathrm{g} / \mathrm{dl})$ & 3,9 & 4,2 & 4,5 \\
Prealbúmina $(\mathrm{mg} / \mathrm{dl})$ & 19,9 & 23,4 & 29 \\
Transferrina $(\mathrm{mg} / \mathrm{dl})$ & 223 & 250 & 275 \\
\hline
\end{tabular}

Solo existieron diferencias en la transferrina en los diferentes estadios, no existieron diferencias significativas en el resto de parámetros.

TABLA V

DISTRIBUCIÓN DE PERCENTILES DE VALORES ANTRO POMÉTRICOS NUTRICIO NALES EN POBLACIÓN DEL ESTUDIO

\begin{tabular}{lccc}
\hline & $\mathrm{P}, 25$ & $\mathrm{P}, 50$ & $\mathrm{P}, 75$ \\
\hline IM C & 20,2 & 22,1 & 24,8 \\
Peso (kg) & 56 & 64 & 71,5 \\
P. tricipital (mm) & 16 & 19 & 22 \\
C. braquial (cm) & 24,5 & 27,2 & 30 \\
C. muscular braquial (cm) & 19,4 & 21,3 & 23,7 \\
\hline
\end{tabular}

IMC (Indice de M asa Corporal).

cunferencia del brazo y la circunferencia muscular del brazo mostraron percentiles 50 por debajo de la normalidad, no así el índice de masa corporal, el peso y el pliegue tricipital (4).

En el análisis de correlación simple entre datos nutricionales antropométricos y bioquímicos con la inmunidad de estos pacientes, solo obtuvimos una correlación positiva entre los valores de CD4 y las proteínas totales $(\mathrm{r}=0,3 ; \mathrm{p}<0,05)$, entre los niveles de CD4 y el PT ( $r=0,4 ; p<0,05)$, y el IMC con los niveles de CD4 ( $r=0,4 ; p<0,005)$, no detectamos otra correlación estadísticamente significativa en este análisis. Realizamos también un análisis de correlación entre los niveles de glucemia y lipidograma con las determinaciones antropométricas, no detectamos ninguna correlación positiva sin embargo si existió correlación entre el número de fármacos antiretrovirales y los niveles de triglicéridos $(r=0,41 ; p<0,001)$.

En un análisis de ANOVA agrupando a los pacientes en función de los diferentes estadios de infección, solo la transferrina presentó valores inferiores estadísticamente significativos en función del estadio VIH del paciente (pacientes estadio C vs A y B) $(262,5+49,2 \mathrm{mg} / \mathrm{dl}$ vs 277,8+87,5 mg/dl; $\mathrm{p}<0,05)$, también encontramos diferencias significativas en los valores de triglicéridos en los mismos estadios de infección $(471,9+501 \mathrm{mg} / \mathrm{dl}$ vs $172,7+105 \mathrm{mg} / \mathrm{dl} ; \mathrm{p}<0,05)$, el resto de parámetros bioquímicos y antropométricos nutricionales no presentaron diferencias significativas en los diferentes estadios de la enfermedad. 


\section{DISCUSIÓN}

Una de las alteraciones nutricionales que más se han descrito en los pacientes con infección por VIH es la tendencia a la desnutrición grave y a la caquexia, por ello esta patología en las poblaciones africanas es denominada la enfermedad adelgazante (slim disease) (5), y en los países occidentales, síndrome constitucional (wasting syndrome) (6). Esta caquexia por VIH, es una de las complicaciones que de manera independiente influye sobre la mortalidad de estos pacientes, suponiendo en muchos casos la única forma clínica de la infección, se estima que un $10 \%$ de los pacientes presentan este cuadro (7). No obstante la desnutrición en el paciente infectado por VIH puede deberse a otras situaciones que no están relacionados con el wasting syndrome; pueden influir alteraciones en la ingesta oral y deglución (8), malabsorción intestinal (9) o incluso alteraciones del metabolismo con aumento del gasto energético basal. En nuestra población de pacientes VIH pudimos comprobar como el peso y el IMC se mantenían dentro de valores normales con una distribución en percentiles correcta; esto puede explicarse en parte debido al alto número de pacientes que estaban recibiendo cuádruple terapia o triple terapia con una media de CD4 del grupo global por encima de 500/uL.

La pérdida de peso uno de los parámetros más accesibles en cualquier consulta, puede facilitar gran información. No obstante en nuestra población de pacientes VIH pudimos comprobar como mantenían un peso e IMC dentro de la normalidad, existiendo una alteración preferentemente en el compartimento proteico muscular, esta última observación está en consonancia con otros estudios que han demostrado una severa reducción del compartimento magro (10). Los trabajos que se han centrado en este problema han demostrado que la pérdida de peso es mayor si existe diarrea acompañante. Otro de los problemas que acompaña a la pérdida de peso es la anorexia. En la práctica diaria se ha detectado que una pérdida de peso lenta (más de 4-5 kg en más de 4 meses) es secundaria a una patología gastrointestinal acompañada de diarrea con mínima afectación del apetito, sin embargo una pérdida acelerada de peso (4-5 kg en menos de 4 meses) con gran afectación del apetito marca la presencia de una enfermedad infecciosa secundaria, por tanto con este simple dato clínico nutricional podemos clasificar a los pacientes VIH en dos grandes grupos de manera diferenciada. No obstante esta percepción del problema ha variado en los últimos años con la incorporación de nuevas terapias (inhibidores de la proteasa), como demuestra las medias de peso e IMC de nuestros pacientes.

Al analizar los parámetros bioquímicos, la mayoría de los estudios demuestran una disminución severa de albúmina. La hipoalbuminemia se ha correlacionado con la supervivencia, de este modo los pacientes con albúmina $<2,5 \mathrm{~g} / \mathrm{d}$ l tienen una supervivencia que no alcanza 20 días, mientras el grupo con albúmina mayor de 3,5 g/dl la media de supervivencia alcanzó casi los 3 años (11). En otros estudios donde se realizó una estratificación por otros factores de riesgo como la edad y la situación inmunitaria del paciente, demostró como el riesgo de mortalidad se multiplicaba por 3,6 en pacientes con valores de albúmina de 3,5 g/dl (10). Otras proteínas como la transferrina, ha demostrado ser útil en la evaluación de desnutrición en estos pacientes (12). En cuanto a la prealbúmina, también ha demostrado su utilidad en detectar la desnutrición subclínica presente en estos pacientes, presentándose como un buen parámetro de control para evaluar la intervención nutricional que realicemos (13). En nuestros pacientes los valores de proteínas nutricionales presentaban medias y distribución de percentiles dentro de la normalidad, solo detectamos una diferencia significativa los niveles de transferrina, con disminución de este parámetro en los pacientes en estadío SIDA. Probablemente esta buena situación nutricional proteica visceral sea también secundaria al alto número de pacientes con terapias triples o cuádruples $(95,1 \%)$ que han demostrado su beneficio en la mejoría de la supervivencia de estos pacientes.

En algunos trabajos se ha señalado que la hipertrigliceridemia presente en estos pacientes puede ser un marcador inicial de la progresión de la infección por el virus y del estado evolutivo de la infección (14). No solo la hipertrigliceridemia es una de las alteraciones descritas en el patrón lipídico en los pacientes VIH diversos autores han demostrado como la hipocolesterolemia es un marcador de evolución clínica desfavorable (15). En nuestro estudio los niveles de triglicéridos fueron superiores en el estadío SIDA y no hubo diferencias en el colesterol, podemos como otro factor implicado además del estadío, el número de fármacos antiretrovirales que toman estos pacientes, ya que en nuestro grupo coincidieron los pacientes con mayor número de tratamientos en el grupo SIDA, existiendo una correlación positiva entre el número de fármacos y los niveles de triglicéridos. En la actualidad se ha descrito como los nuevos tratamientos antiretrovirales presentan como efecto secundario una profunda alteración del metabolismo lipídico y de los hidratos de carbono, estas alteraciones como ha demostrado recientemente nuestro grupo pueden ser reversibles con un tratamiento adecuado (16).

Es necesario tener en cuenta que existen una serie de factores que a veces son olvidados y que pueden influir en el estado nutricional de los pacientes con infección por VIH, estos son factores psicosociales como el entorno familiar, laboral y afectivo de estos pacientes (17).

En conclusión, el estado nutricional de los pacientes VIH en nuestro estudio es bueno presentando solo una depleción del compartimento proteico muscular. La transferrina se ha mostrado como un parámetro nutricional disminuido en los estadios avanzados. La hipertrigliceridemia existente en estos pacientes parece secundaria al tratamiento antiretroviral.

\section{Bibliografía}

1. Weekly epidemiological record 1999. 26 Jun. N 26, 195-198. World Health Organization. Geneva.

2. Mataix J, Llopis J. Evaluación del estado nutricional. En: Nutrición y salud pública: Métodos, bases científicas y aplicaciones. Barcelona. De. Masson, 1995;73-89.

3. Blackburn GL, Bristian BR, Maini BS. Nutritional and metabolic assessment of the hospitalized patient. En: Nutrition assessment anthology. New York. De. ASPEN, 1991:1-13.

4. Frisancho AR. News norms of upper limb fat and muscle areas for assessment of nutritional status. Am J Clin Nutr 1981;34:540-545. 
5. Polo Rodriguez RM. Nutrición en el paciente VIH positivo. Seisida 1996:7: 521-524.

6. Shenk A. Resting energy expenditure, weight loss, and altered body composition in HIV infection. Nutrition 1996;12:595-601.

7. HIV/AIDS Surveillance in Europe. First quarterly Report 1998 n 57. European Centre for the epidemiological Monitoring of AIDS. WHOEU collaborating Centre on AIDS. Saint Meurice, France

8. Greene JB. Clinical approach to weight loss in the patient with HIV infection. Gastroenterol Clin North Am 1988:17:573-586.

9. Kotler DP. Malnutrition in HIV infection and AIDS. AIDS 1989;3:175180

10. Guenter P, Muurahein N, Simons G, Kosos K, A, Cohan Gr, Rudenstein $\mathrm{R}$, Turner JL. Relationship between nutritional status, disease progression, and survival in HIV infection. J Acquir Immune Defic Syndr 1993;6:1130-1138.

11. Chlebowski RT, Grosvenor MB, Bernhard NH, Morales LS, Bulcavage LM. Nutritional status, gastrointestinal dysfunction, and survival in patients with AIDS. Am J Gastroenterol 1989; 84: 1288-1231.

12. Shetty PS, Watrasiewicz KE, Jung RT, James WPT. Rapid turnover transport proteins: an index of subclinical protein-energy malnutrition. Lancet 1979;4:230-232.

13. Shetty PS, Watrasiewicz KE, Jung RT, James WPT. Rapid turnover transport proteins: an index of subclinical protein-energy malnutrition. Lancet 1979; 4: 230-232.

14. Gomez Sirvent JL, Alonso Socas MM, Santolaria Fernandez FJ, Gonzalez Reimers CE, Rodriguez Moreno F, Essardas Daryanani H. Prognosis value of triglyceride levels in early HIV infection. AIDS 1994; 8: 1740-1741.

15. Dursum SM, Reveley MA. Hypocholesteronemia and human immunodeficiency virus 1 (HIV-1) infection. Am J Med 1995; 98: 518-521.

16. De Luis DA, Aller R, Bachiller P, Tortosa JI. Reversibilidad de hipertrigliceridemia intensa secundaria a indinavir con fenofibrato micronizado. Med Clin (Barc) 1999; 113: 716-717.

17. Sánchez alvarez MC, Gomez Ramos MJ, Cano Sanchez A, Pacheco Guevara R, Nicolas Hernadnez M, García Alberola A. Evolución del estado nutricional de pacientes VIH-sida. Efecto de su situación socioeconómica y del consejo dietético. An Med Interna (Madrid) 1998; 12: $627-632$. 